\title{
A Self-Sealing Spray Nozzle for Aerosol Drug Delivery
}

\author{
Torben Sebastian Last ${ }^{\circledR}$, Niclas Roxhed $\left.{ }^{(}\right)$, and Göran Stemme ${ }^{(\circledR)}$, Fellow, IEEE
}

\begin{abstract}
Chronic lung disease is commonly treated using portable inhaler systems. By design, these inhalers come into repeated contact with the mouth region of patients and are therefore subject to bacterial contamination and ingrowth. To enable the safe delivery of an aerosol from a drug reservoir of such an inhaler, the reservoir needs to be protected from pathogens. Here we demonstrate a self-sealing aerosol spray nozzle, a system that seals each spray orifice on nozzle level in its default closed state and sprays a liquid Rayleigh-jet upon actuation. The sealing mechanism is realized by placing a thin membrane with spray orifices on top of a valve seat. The membrane behavior and spray performance of the self-sealing spray chip were found to be identical to an equivalent non-sealing design. This sealing system may enable the safe reuse of spray chips for multiple spray operations over an extended period, lowering the cost of treatment while increasing patient compliance.

[2019-0252]
\end{abstract}

Index Terms-BioMEMS, drug delivery, parylene, fluidic microjet, microfluidics.

\section{INTRODUCTION}

C HRONIC lung diseases such as asthma or chronic obstructive pulmonary disease (COPD) continue to be among the leading causes of illness worldwide [1]. The world health organization predicts COPD to be the fourth leading cause of early death by 2040 [2]. Treatment is applied by the inhalation of aerosols, nebulized powders or liquids, administered repeatedly over an extended period [3]. The dominant portable inhaler systems on the market today are pressurized metered-dose inhalers (pMDI) and dry powder inhalers (DPI) [3], which aerosolize liquid and powdered drug formulations, respectively. Efforts to increase the drug delivery efficiency of portable liquid inhalers have lead to the development of so-called soft mist inhalers (SMIs). SMIs present distinct advantages over other portable inhaler types. Namely, they are propellant-free, feature an active aerosol generation which enables a comfortable slow inhalation pattern for the patient, and allow for excellent control of the aerosol particle sizes [4], [5]. SMIs can be made using a springloaded system to create an aerosol by forcing a liquid drug through spray orifices in a thin membrane at high pressure. A liquid column forms, which breaks up into droplets of a defined size

Manuscript received November 30, 2019; revised January 9, 2020; accepted January 21, 2020. This work was supported by SHL Group AB. Subject Editor C. Ahn. (Corresponding author: Torben Sebastian Last.)

The authors are with the Department of Micro and Nanosystems, School of Electrical Engineering, KTH Royal Institute of Technology, 10044 Stockholm, Sweden (e-mail: tslast@kth.se).

Color versions of one or more of the figures in this article are available online at http://ieeexplore.ieee.org.

Digital Object Identifier 10.1109/JMEMS.2020.2969611 due to surface tension [5], forming a Rayleigh-jet. The droplet size is dependent on the diameter of the liquid column which is defined by the spray orifice diameter [5]. Rayleigh-jetbased inhalers feature high throughput monodisperse droplet generation, aerosols that have a narrow particle size distribution [6], [7]. This type of aerosolization allows for simple, light-weight, portable, low-cost and high-performance inhalers [5].

However, the microbial safety of SMI devices is so far lacking in comparison to other portable inhalers. DPIs store drugs in the dry state and sometimes through blister systems, in which each delivery dose is packaged and sealed separately [6]. pMDIs are currently the prevalent liquid inhaler system and use a dosing-valve to seal the spray element from the liquid drug supply, which allows for multi-dose treatment [8]. Similar no-return valve systems have made their way to SMI devices [9]. But even with the added complexity of a separate valve system, the actual aerosolization unit and the liquid path to the valve are exposed to the environment.

In the case of Rayleigh jet-based SMIs, the spray orifices are constantly exposed, allowing bacterial ingrowth and contamination of the spray nozzle [10]. Hence, wide adoption of these low inspiratory effort inhalers has been hampered by simpler, multi-use DPIs, which require precise forced inhalation at a sufficient flow rate. However, such an inhalation can be difficult for patients suffering from lung disease to achieve [11]

We recently demonstrated the concept of a self-sealing spray nozzle [12]. Here we expand on this work, describing a self-sealing spray nozzle system which seals each spray orifice against bacterial ingrowth by geometric constraint. Our sealing system is intended to effectively prevent bacterial ingrowth into the drug reservoir, thereby enabling the use of SMIs in multi-use inhaler systems, a market segment so far dominated by DPIs.

\section{PRINCIPLE AND DESIGN}

The self-sealing spray chip is a dynamic system that relies on an applied differential pressure to deflect a spray-membrane off a valve seat and open a channel, which the fluid drug has to pass, before reaching the spray orifices. The sealing system rests in a normally closed position and can be opened on demand for spray operation using an applied differential pressure as illustrated in Fig. 1.

The spray chip features two distinct layers with fluidic function; a spray and a sieve layer. The sieve layer filters the 
a) closed

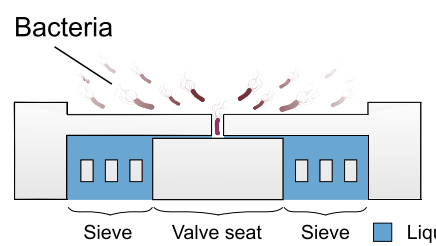

b) open

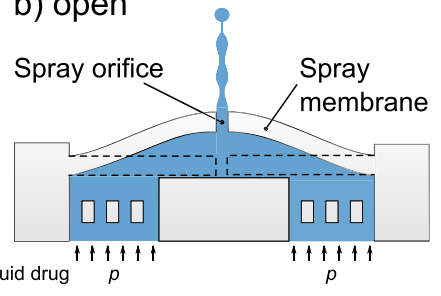

Fig. 1. Schematic drawing showing the self-sealing spray nozzle in a) closed and b) open state. Without applied pressure, the system rests in its default closed state and prevents bacteria from passing the liquid channel between valve seat and spray membrane. Upon pressurization the spray membrane deflects, and the sealing mechanism opens, creating an aerosol for inhalation.

liquid to be sprayed before reaching the spray layer, where it is aerosolized, as shown in Fig. 2 a. Sieving the liquid prevents clogging of spray orifices by particles contained in the drug solution, which may encompass suspensions. In our current design sieve orifices have the same diameter as spray orifices: $2 \mu \mathrm{m}$. The sieve layer further incorporates a valve seat, which together with the spray membrane forms the sealing mechanism. The spray and the sieve layer are bonded using Parylene-C as a bonding layer, which also acts as a spacer between the valve seat and the spray membrane. To have the function of a microbial barrier, the channel between the valve seat and the spray nozzle membrane needs to be constricted in its smallest dimension to less than $300 \mathrm{~nm}$ in the non-pressurized state. Such gaps have previously been shown to prevent the ingrowth of motile E. Coli bacteria in silicon-based devices [13], and are also close to the $200 \mathrm{~nm}$ filter pore size routinely used in sterilization processes of liquids in the pharmaceutical industry [14]. Fig. 2b shows a photograph with the spray chip $(3.8 \mathrm{~mm}$ by $1 \mathrm{~mm})$ in size comparison to a matchstick. The sealing mechanism comprises a $2.5 \mu \mathrm{m}$ thick silicon membrane located on top of a $30 \mu \mathrm{m}$ wide valve seat. The rectangular spray membrane has a width of $150 \mu \mathrm{m}$ and a length of $2 \mathrm{~mm}$. 100 spray nozzles of $2 \mu \mathrm{m}$ diameter are pitched $10 \mu \mathrm{m}$ apart on the spray membrane. Fig. $2 \mathrm{c}$ shows a microscope picture of a self-sealing spray chip with the nozzle row centered on top of the valve seat.

\section{FABRICATION}

Our spray chip design has been developed with mass manufacture in a typical microfabrication facility in mind. We only use standard materials and processesing steps, which are available at scale. The sieve and spray layers are manufactured from $100 \mathrm{~mm}$ silicon on insulator (SOI) wafers with a device layer thickness of $2.5 \mu \mathrm{m}$ and a handle layer thickness of $300 \mu \mathrm{m}$. Fig. 3 a illustrates the fabrication. A $300 \mathrm{~nm}$ thick layer of $\mathrm{SiO}_{2}$ was deposited as a hard mask on the device layer using plasma-enhanced chemical vapor deposition (PECVD). I-line vacuum contact lithography was used to define orifices with diameters of $2 \mu \mathrm{m}$ on the frontside of the wafers. The resulting frontside pattern was etched into the hard mask by means of reactive ion etching (RIE). Using the hard mask, the silicon device layer was structured using RIE. A $300 \mathrm{~nm}$ layer of PECVD oxide was deposited on the etched device layer as protection during further processing. Then a $2 \mu \mathrm{m}$ $\mathrm{SiO}_{2}$ hard mask was deposited on the handle layer of the wafers using PECVD. This hard mask is used for structuring the handle layer of the wafers. The handle layer hard mask was patterned using contact lithography and RIE of $\mathrm{SiO}_{2}$. The rectangular valve seat is defined by etching two trenches for each chip on the sieve wafer. The distance between the trenches then defines the valve seat. We fabricated structures with a valve seat width of $30 \mu \mathrm{m}$. For the spray nozzle wafer, a single trench was etched into the backside of each spray chip, to geometrically define the spray membrane. The buried oxide of the SOI wafers was removed using $50 \%$ hydrofluoric acid, releasing the membranes on the device layer. All further processing was performed on freestanding silicon membranes. Parylene- $\mathrm{C}$ was deposited as a bonding layer for wafer-level thermocompression bonding. Parylene-C is a chemically inert polymer that is deposited from the gas phase. It is well documented in bonding behavior [15]-[17] and holds the highest biocompatibility rating for plastics (ISO 10993 USP VI) [18]. To enhance Parylene adhesion to silicon, both wafers were immersed for $30 \mathrm{~min}$ in a solution composed of $0.5 \mathrm{v} / \mathrm{v} \%$ Merck A-174 silane solution, $49.75 \mathrm{v} / \mathrm{v} \%$ distilled water and $49.75 \mathrm{v} / \mathrm{v} \%$ isopropanol. After adhesion treatment, $800 \mathrm{~nm}$ of Parylene-C was deposited on the device layer of the sieve wafer using an SDS Labcoater 2.

Adhesive bonding was performed using either $150 \mathrm{~nm}$ or $800 \mathrm{~nm}$ of Parylene on the device layer side of the spray nozzle wafer. The Parylene thickness on the sieve layer was kept to $800 \mathrm{~nm}$ for all tests. When the Parylene is selectively removed from the spray nozzle membrane, the gap height before bonding between valve seat and membrane is defined entirely by the Parylene thickness on the spray nozzle side. We saw no decrease in bonding performance by decreasing the Parylene thickness and consequently all fluidic measurements presented in this paper were performed with $150 \mathrm{~nm}$ of Parylene on the nozzle wafer. After Parylene deposition, a positive photoresist was spincoated on top of the Parylene layer. We used multiexposure soft-contact lithography to reduce the heat generated by the crosslinking photoresist, which otherwise leads to Parylene degradation. The Parylene layer was etched using oxygen plasma in an STS Multiplex etcher.

Bonding on wafer-level was performed using a Süss Microtec $\mathrm{CB} 8$ at $3.5 \mathrm{kN}$ and $250^{\circ} \mathrm{C}$ for $30 \mathrm{~min}$. A crosssection of a valved spray chip after bonding is shown in Fig. 3 b, with a measured gap height of about $260 \mathrm{~nm}$ between spray membrane and valve seat. The bond alignment was verified using microscopy to ensure that spray orifices align with the valve seat after bonding as shown in figure $2 \mathrm{c}$.

Non-sealing chips were manufactured from a single SOI wafer featuring the same membrane dimensions, and spray orifice layout as the self-sealing chips. This wafer has been subject to the same processing steps involved in manufacturing the self-sealing spray chips. We evaluated the spray orifice diameters of five different chips of self-sealing and non-sealing configuration, respectively. We measured 20 orifice diameters per chip, resulting in 100 measurements per chip configuration. 
a)

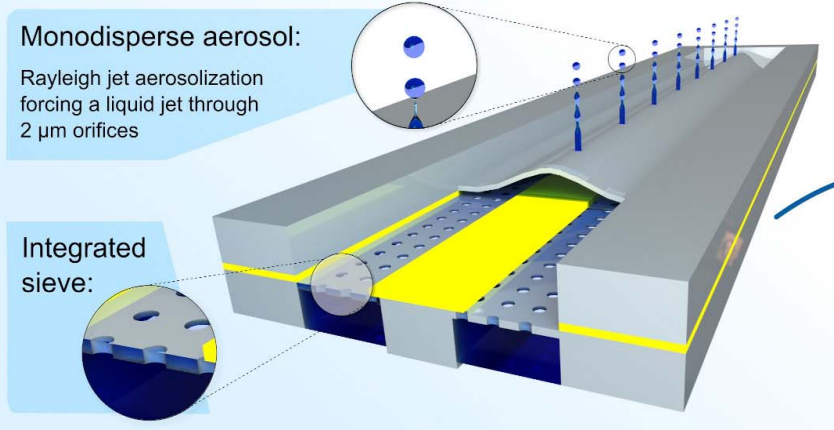

b)

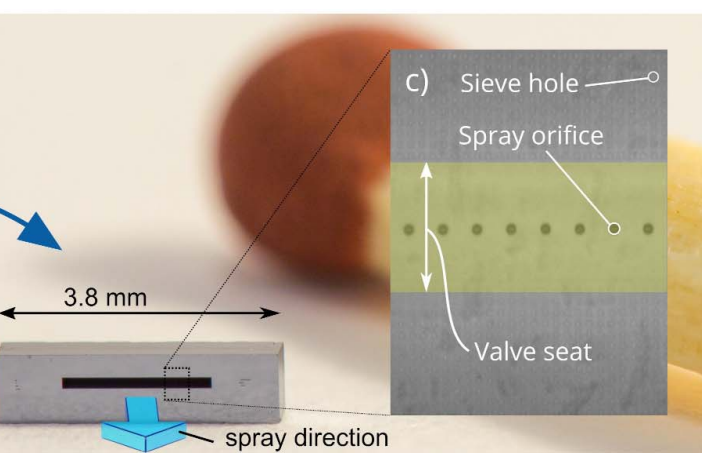

Fig. 2. a) Rendered image of the self-sealing spray chip. b) Frontside picture of a spray chip with the spray direction indicated by the blue arrow, and a matchstick head in the background for size comparison. c) Microscope picture showing a topside view using both front- and backside illumination with the nozzle holes located on the valve seat (colored). The sieve orifices can be seen through the spray membrane.
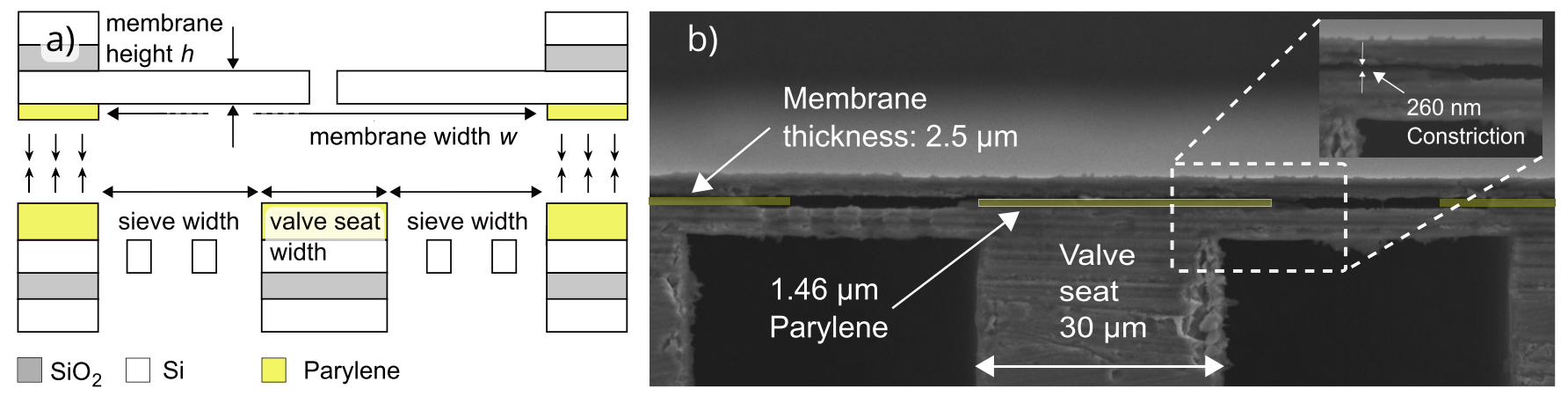

Fig. 3. a) Fabrication schematic showing a nozzle and a sieve layer before the bonding step. b) SEM cross-section picture of a self-sealing spray chip. The Parylene layers are highlighted in yellow in the overview. The constriction between valve seat and silicon membrane is $\sim 260 \mathrm{~nm}$, measured using a Hitachi S3400 N. Two different Parylene layers were evaluated $(800$ and $150 \mathrm{~nm}$ ), a $800 \mathrm{~nm}$ sample is shown here. Parylene compression during the bonding leads to a measured combined Parylene height of $1.46 \mu \mathrm{m}$.

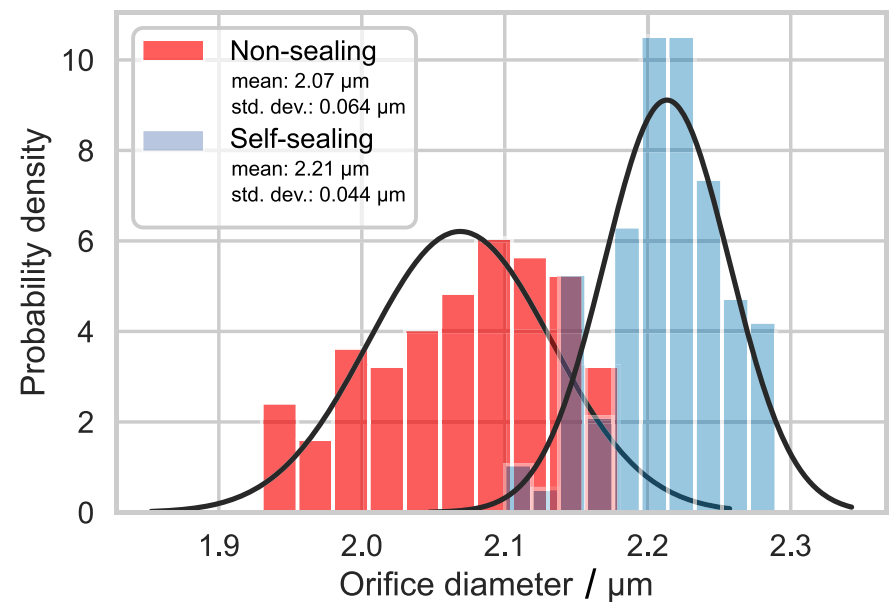

Fig. 4. Distribution plot showing orifice diameters for self-sealing and non-sealing configurations $(n=100)$ of spray chips as measured using a Hitachi S3400 N. Black lines show a fit to a GauSSian distribution, from which mean diameters and standard deviations were extracted.

A distribution plot of the measurement data with mean values and standard deviation is shown in Fig. 4.

Fig. 4 shows a difference of $144 \mathrm{~nm}$ in mean diameter between self-sealing and non-sealing spray chip configurations, due to the wafers coming from different manufacturing batches. Tools and process parameters were identical for those two batches.

\section{Methods}

\section{A. Membrane Deflection and Rupture Testing}

To evaluate the deflection and rupture pressure of silicon membranes we used a setup in which we pressurized the chip using nitrogen from a 300 bar nitrogen gas bottle. The spray chips were glued to an aluminum frame using cyanoacrylate, and the aluminum frame was connected with an O-ring to an aluminum piece with a $1.5 \mathrm{~cm}^{3}$ cavity forming the pressurization chamber. The pressure-dependent membrane deflection was measured using a white light interferometer (Wyko NT 9300). A pressure regulator on the nitrogen gas bottle combined with a pressure gauge was used to achieve the desired pressure in the pressurization chamber. We validated the setup to be capable of holding pressures of up to $40 \mathrm{bar}$, which was the maximum rating of the pressure sensor used in the system.

\section{B. Fluidic Operation Testing}

Spray chips were glued to a custom plastic package and pressurized using a pneumatic actuator (Festo ADN-50-80) acting on a $20 \mathrm{~mL}$ plastic syringe filled with distilled DI water. The spray chip package was connected to the syringe via PEEK tubing. Using a pneumatic actuator allows for precise and fast pressure ramping, similar to how the spray chips encounter pressure in a spring-loaded inhaler system. 
a)

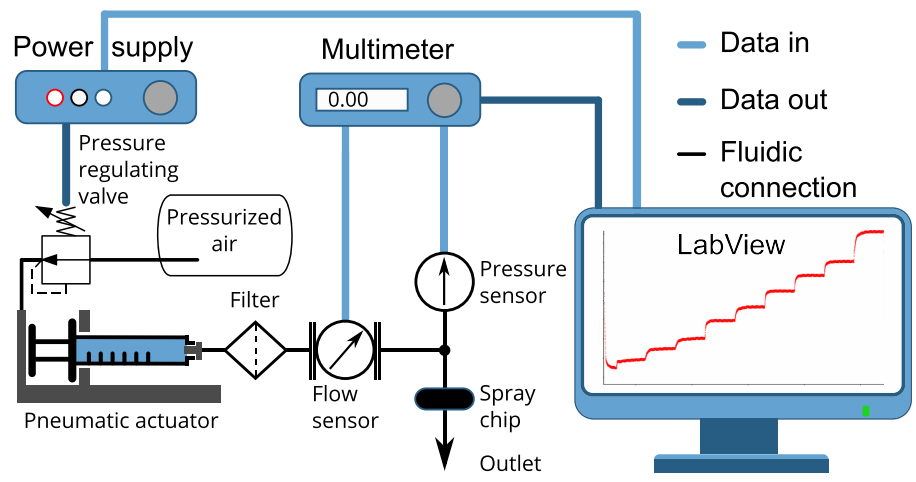

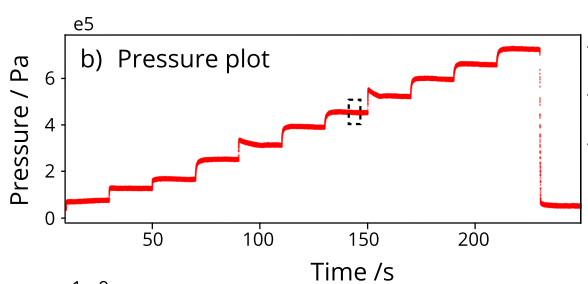
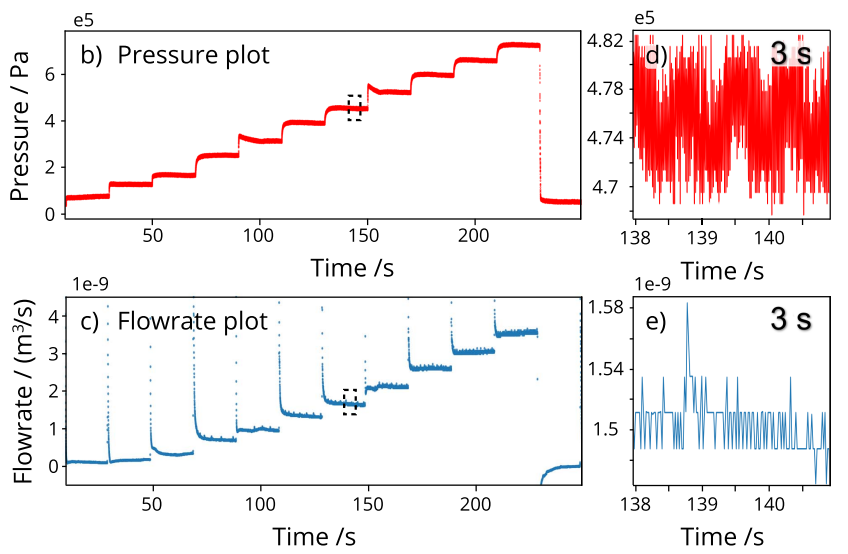

Fig. 5. a) Schematic drawing of the fluidic measurement setup. A complete set of measurement steps with a holdtime of $20 \mathrm{~s}$ is shown in b) for the pressure and c) for the flow rate. Mean values of pressure and flow rate were taken by averaging datapoints over $3 \mathrm{~s}$ segments as shown in d) and e).

Using a pressure regulating valve (Festo VPPE), we regulate the force exerted by the pneumatic actuator on the syringe. The pressure regulating valve is voltage-controlled using LabView as illustrated in Fig. 5 a.

A syringe filter with a pore size of $0.45 \mu \mathrm{m}$ was placed downstream of the syringe to prevent debris from the syringe's plunger mechanism from entering the measurement setup. We exclusively used 1/16 inch PEEK tubing for all fluidic connections and Upchurch 10-32 standard cone port connectors between components to achieve leak-tight connections. A Variohm EPT 310010 bar pressure sensor was mounted in an acrylic block fitted with Upchurch connections for the flow inlet and outlet. The acrylic components were cleaned using isopropanol in an ultrasonic bath before being installed in the measurement setup. Afterward, the system was thoroughly flushed with DI water by actuating the syringe. The flow rate was measured using a Sensirion (ASL 1600-10) sensor upstream of the spray chip, as illustrated in Fig. 5. The flow sensor signal was sampled at $50 \mathrm{~Hz}$. The pressure sensor was connected to an HP 34401A Multimeter, sampled at $250 \mathrm{~Hz}$. Data from the pressure and flow sensors was recorded using a LabView script.

We compared self-sealing and non-sealing chips in this setup. Non-sealing chips were measured without a sieve layer. Notably, the sieve layer features 15624 sieve orifices $\left(n_{s}\right)$ compared to 100 spray orifices $\left(n_{o}\right)$ of the same diameter. The influence of the sieve on the combined fluidic resistance may therefore be estimated by $n_{o} / n_{s}$ to $0.64 \%$, taking the values from TableI. This is smaller than the effect of the mean orifice diameter deviation between the tested self-sealing and non-sealing configurations, and we have therefore not compensated for sieve resistance in our testing. Spray chips were pressurized in discrete $20 \mathrm{~s}$ steps. A full pressurization cycle of the actuating pressure is shown in Fig. $5 \mathrm{~b}$, featuring ten datapoints for each respective flow rate and pressure combination, from which we calculate the mean value and the standard deviation of the measurement. The fluidic resistance was calculated by taking the mean value of the flow rate and pressure for a $3 \mathrm{~s}$ segment of the respective pressure step, and then averaging over the ten data points.

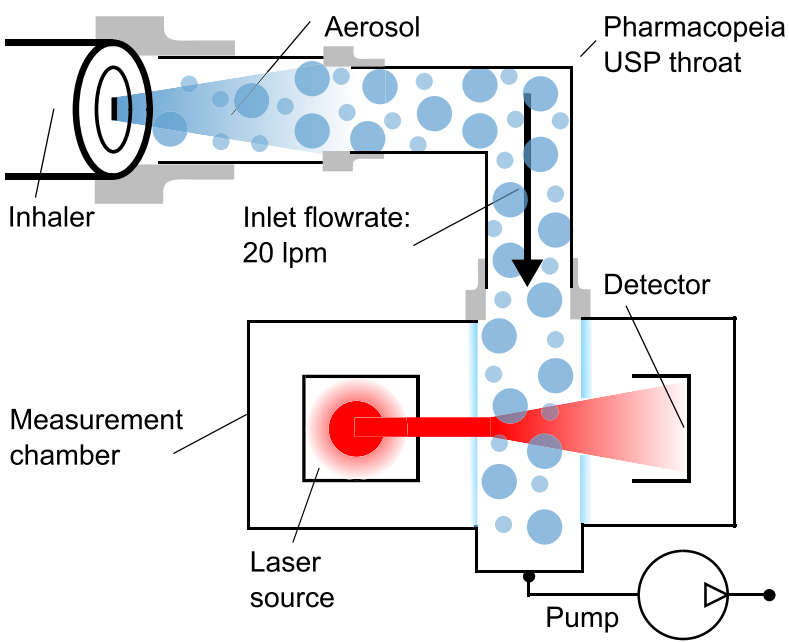

Fig. 6. Schematic drawing showing the laser diffraction measurement setup using the Malvern Spraytec. The aerosol generated by the spray chip passes the USP throat with an inlet flow rate of $20 \mathrm{~L} \mathrm{~min}^{-1}$, before entering the measurement chamber.

\section{Spray Testing}

Spray tests were conducted with the spray chips mounted in an inhaler system (Pharmaero ADI), in which the spray chip was pressurized using an internal spring providing 25 bar of pressure. We assembled spray chips in a custom plastic holder which in turn was mounted in the inhaler system. For spray testing, we used a $9 \mathrm{mg} \mathrm{mL}^{-1}$ sodium chloride solution. Each spray actuation aerosolizes $50 \mu \mathrm{L}$ of liquid. A United States Pharmacopeia (USP) induction port was used as a throat model and installed upstream of the measurement chamber. The aerosol was fed into the measurement instrument with a fixed $20 \mathrm{~L} \mathrm{~min}^{-1}$ inlet flow. The aerosol particle size distribution was measured by laser diffraction analysis in a Malvern Spraytec instrument, as illustrated in Fig. 6. The Malvern Spraytec records a time-resolved diffraction pattern, representative of the aerosol entering the lung.

\section{Results And Discussion}

We designed our self-sealing spray chip to seal each spray orifice separately on top of a valve seat. Thereby we eliminate 

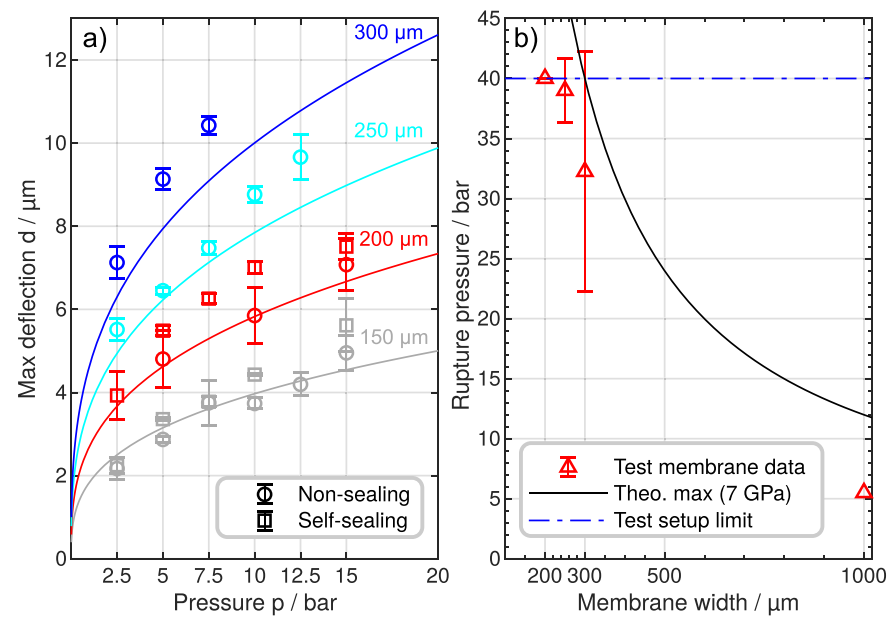

Fig. 7. a) Deflection measurements of self-sealing and non-sealing spray chip configurations with different membrane widths. Solid lines indicate expected theoretical values from eq.1. b) Measured rupture pressure of rectangular silicon membranes, evaluated against eq. 3. Error bars show standard deviation between measurements where indicated.

any dead volume in the design which might be contaminated by pathogens. Further, since all spray orifices are aligned in a straight line, the membrane deflection does not affect spray direction. The die size of the device is currently 3.8 by $1 \mathrm{~mm}$ and may be miniaturized even further. Such a small chip size can potentially allow for cost-effective production of spray chips at an industrial scale.

\section{A. Mechanical Membrane Behavior}

The maximum deflection of the nozzle membrane is described by a non-linear function of membrane geometry and pressure. For a square membrane, which is clamped on four edges and subjected to uniform pressure, the maximum deflection $d$ is given by:

$$
d=0.318 w \sqrt[3]{\frac{p w}{E h}},
$$

and depends on the applied differential pressure $p$, membrane width $w$, Young's modulus of silicon $E$, and membrane thickness $h$ [19]. The measured deflections of our nozzle membranes are shown in Fig. $7 \mathrm{a}$ and compared to the theoretical values given by eq. 1 . For a membrane width of $150 \mu \mathrm{m}$ a membrane deflection of $2.5 \mu \mathrm{m}$ is reached at differential pressures of 4-5 bar.

Based on theoretical estimations a channel height between valve seat and spray membrane of $2.5 \mu \mathrm{m}$ would lead to a fluidic resistance significantly lower than the spray orifice resistance. At a spray pressure of 25 bar the sealing mechanism should be completely open, and the fluidic resistance then is solely defined by the spray orifice resistance.

The generation of Rayleigh-jets is governed by a combination of inertia and surface tension [20]. If the kinetic energy of the fluid leaving the nozzle is high enough to form a standing liquid column capillary breakup is induced and the droplet generation changes from the dripping to the jetting regime, which is the favorable operating regime for monodisperse droplet generation [5]. The differential pressure required to initiate a Rayleigh-jet from a nozzle depends on nozzle geometry and liquid viscosity [5]. Typical fluid viscosities range from $0.01 \mu \mathrm{Pa}$ s to $1 \mathrm{mPa}$ s, i.e spanning five orders of magnitude [21]. To enable the use of the same spray chip for a relevant variety of fluid viscosities of different liquid drugs, the operating pressure range has to be sufficiently large. To safely reach the jetting regime using our spray chips, we used an operating pressure of $25 \mathrm{bar}$, a pressure the spray membrane has to withstand without rupturing.

The rupture pressure is determined by geometry, support conditions and the yield strength $\sigma_{\text {yield }}$ of the membrane material. In the following we use a value of $130 \mathrm{GPa}$ for the Young's modulus of silicon, as recommended in [22]. The total stress of a double-clamped square membrane according to [23] can be written as:

$$
\sigma_{\text {tot } .}=1.442 \sqrt[3]{\frac{p^{2} w^{2} E}{h^{2}}} .
$$

Membrane rupture occurs when $\sigma_{\text {tot }} \geq \sigma_{\text {yield }}$. We use a value of $7 \mathrm{GPa}$ for the yield stress of silicon [24]. The rupture pressure

$$
p_{r}=\sqrt{\left(\frac{\sigma_{y i e l d}}{1.442}\right)^{3} \frac{h^{2}}{w^{2} E}}
$$

can be calculated by redistributing terms from eq. 2 and substituting the yield stress $\sigma_{\text {yield }}$ for $\sigma_{\text {tot. }}$. The measured break pressures of our silicon membranes are illustrated in Fig. $7 \mathrm{~b}$ and correspond well to theoretical expectations. Monocrystalline silicon is an ideal mechanical material, free of defects in its crystalline structure [24]. The spray membrane does not encounter stress levels higher than half the yield stress at operating pressure. During our testing, we have not encountered a single spray membrane out of ca. 75 tested devices to rupture at operating pressure. Further, none of the tested $150 \mu \mathrm{m}$ membranes ruptured at the maximum pressure of the test setup of 40 bar, almost twice the operating pressure.

\section{B. Fluidic Behavior}

In a first-order approximation, the channel between the valve seat and the spray membrane may be considered to be rectangular. The resulting hydrodynamic fluidic resistance under laminar flow conditions is then given by

$$
R_{c}=\frac{12 \eta l}{w h_{c}^{3}},
$$

where $h_{c}$ is the channel height, $\eta$ is the fluid viscosity, $l$ the channel length and $w$ the channel width [25]. The values used for the calculation of hydrodynamic resistance are summarized in Table I. The fluidic resistance of a spray orifice with an aspect ratio between orifice height $h_{o}$ and orifice diameter $d$ of $h_{o} / d \leq 2$ can be approximated by:

$$
R_{o}=\left(1+\frac{16 h_{o}}{3 \pi d}\right)\left(\frac{24 \eta}{d^{3}}\right)
$$

when $R e \leq 1$ (creeping flow conditions) as first demonstrated by Dagan et al. [26]. Comparing $R_{c}$ for a $30 \mu \mathrm{m}$ long channel 
TABLE I

HydRodynamic RESistance CALCUlation VAlues

\begin{tabular}{llll}
\hline Parameter & Symbol & Value & Unit \\
\hline Dynamic viscosity of $\mathrm{H}_{2} \mathrm{O}[21]$ & $\eta$ & 1 & $\mathrm{mPas}$ \\
Valve seat length & $l_{v}$ & 30 & $\mu \mathrm{m}$ \\
Valve seat width & $w_{v}$ & 2 & $\mathrm{~mm}$ \\
Membrane width & $w$ & 150 & $\mu \mathrm{m}$ \\
Sieve and spray orifice height & $h_{o}$ & 2.5 & $\mu \mathrm{m}$ \\
Sieve and spray orifice & $d$ & 2 & $\mu \mathrm{m}$ \\
diameter & & & \\
Nr. of spray orifices & $n_{o}$ & 100 & - \\
Nr. of sieve orifices & $n_{s}$ & 15624 & - \\
\hline
\end{tabular}

with an array of 100 spray orifices of $2 \mu \mathrm{m}$ diameter using eq. 5, one finds that a membrane deflection of more than $2.5 \mu \mathrm{m}$ is needed to reduce the channel resistance to be significantly lower than the orifice resistance $\left(10 \% R_{o}\right)$.

The fluidic resistance of a single orifice $R_{S}$, can be derived from the total nozzle array resistance $R_{t o t}$ of a given nozzle array with $n_{0}$ orifices using:

$$
R_{S}=\frac{R_{t o t}}{n_{o}}=\frac{\Delta p}{n_{0} Q_{t o t}}
$$

with the applied differential pressure $\Delta p$, the number of spray orifices $n_{o}$ and the flow rate through the nozzle array $Q_{t o t}$. The single orifice resistance allows for easy comparison between different nozzle array configurations. Fig. 8 a shows the measured fluidic resistance for self-sealing and non-sealing spray chips, respectively.

It can be seen that the sealing mechanism opens gradually with applied differential pressure, resulting in a decrease in hydrodynamic channel resistance. At pressures below 2 bar, the variation of measurement is large, as the flow rate is very low $\left(2 \mu \mathrm{L} \mathrm{min}^{-1}\right)$ and close to the minimum that can be measured with the system. Nevertheless, the fluidic resistance of the self-sealing spray chips at 0.5 bar is around 20 times higher than that of non-sealing spray chips. In an inhaler system, no pressure is acting on the sealing system, leading to a closed state. At differential pressures above 4 bar, the fluidic resistance of the self-sealing spray chip is approaching the ideal analytical value, as does the measured value for a non-sealing spray chip. That is one-fifth of the operating pressure of the inhaler system. We measured fluidic resistance in gradual steps. However, in a spring-loaded inhaler the transition from closed ( 0 bar) to open state ( $25 \mathrm{bar}$ ) is rapid, and takes roughly $200 \mathrm{~ms}$.

In our testing, we have dispensed more than $50 \mathrm{~mL}$ of clean DI-water per tested self-sealing spraychip without noticeable clogging. However, sieving performance will vary with the liquid drug used in the system. Additionally, more advanced filter elements could be incorporated to prevent premature clogging of the mono-sized sieve structure by big particles, if needed.

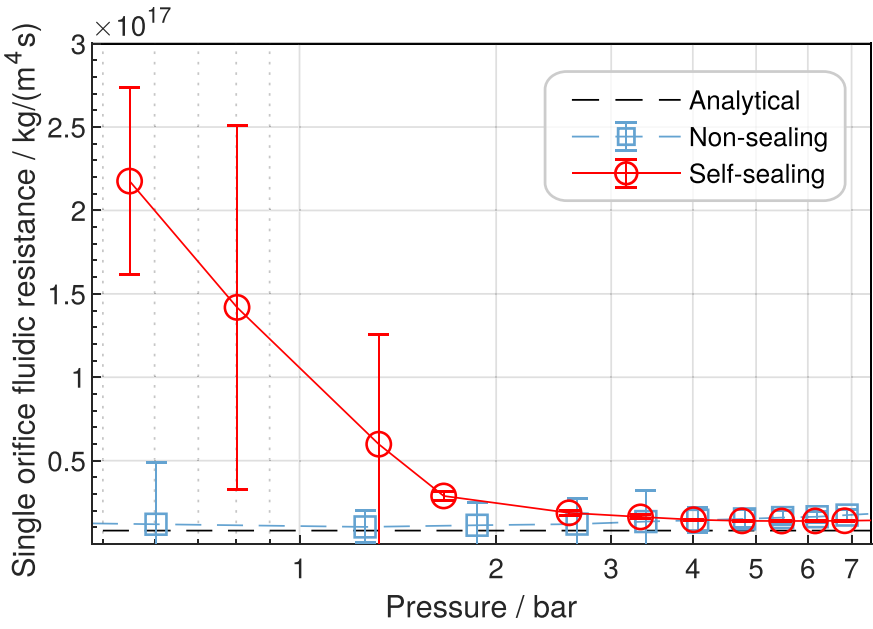

Fig. 8. Measured fluidic resistance of self-sealing and non-sealing spray chips, depending on applied differential pressure and compared to the analytical resistance of a $2 \mu \mathrm{m}$ nozzle of eq. 5 .

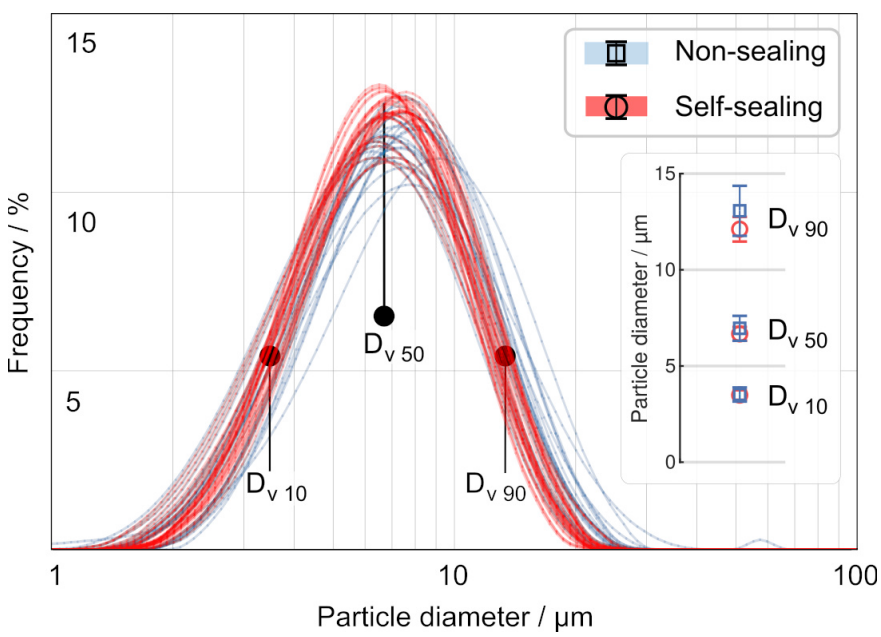

Fig. 9. Measured volumetric particle size distribution of self-sealing and non-sealing spray chips mounted in an inhaler system and pressurized at 25 bar ( $\mathrm{n}=30$ for each configuration: 10 actuations of three chips). Spray chips have an array of $1002 \mu \mathrm{m}$ nozzles. The produced aerosol is identical within the standard deviation of the dataset for the three indicated $D_{v}$ points, indicating that the sealing system has no influence on the spray performance of the spray chip.

\section{Spray Testing of Self-Sealing and Non-Sealing Chips}

We measured the aerosol particle size distribution of three self-sealing and three non-sealing spray chips using laser diffraction. Laser diffraction measurement is a standardized (ISO 13320), robust and reliable [27] method for acquiring time-resolved particle size distributions of aerosols and regularly used as a standard technique in the pharmaceutical industry [28]. To measure a representative aerosol sample, aerosol particles need to be consistently dispersed [29]. If a sample is not fully dispersed in the gas flow, this will negatively affect the reproducibilty of the measurement [28]. We actuated each spray chip ten times using a spring-loaded inhaler at 25 bar, and get consistent results which are shown in Fig. 9. The measurements are presented in a volume distribution plot, showing the contribution of specific particle sizes to the total volume of the aerosolized drug. We analyzed the 
spray distribution dataset at three distinct points; $D_{v} 10, D_{v} 50$ and $D_{v} 90$ (diameter at which $\mathrm{x} \%$ of the aerosol volume have particles smaller than the indicated value). We then compared the different spraychip configurations.

Although the orifice diameters between self-sealing $(2.21 \mu \mathrm{m})$ and non-sealing $(2.07 \mu \mathrm{m})$ chips differ slightly, and the non-sealing spray chips feature no sieving structure, the resulting aerosol size distributions are identical within the standard deviation of the dataset for all three $D_{v}$ points, visualized in the inset of Fig.9. Based on the volumetric particle size distributions the sealing mechanism does not influence spray performance. It would be interesting to further look at single droplet formation at the nozzle exit in future work. Clogging and other long-term degradation effects of self-sealing spray chips will also need to be investigated further.

\section{CONCLUSION}

We present a self-sealing aerosol nozzle valve for Rayleigh-jet based inhalers, that does not impede the fluidic behavior of the spray chips at operating pressure while being closed in the default, non-pressurized state. As we seal on nozzle orifice level, there is no dead volume in the spray chip accessible to pathogens. In a fluidic setup, we demonstrated that lifting the spray membrane off the valve seat gradually reduces fluidic resistance, and the device behaves identically to a non-sealing spray chip configuration at a differential pressure larger than 4 bar, one-fifth of the operating pressure. We characterized the produced aerosol from self-sealing and non-sealing spray chips and found them to be identical. This sealing system could lead to safe, high-performance inhalation devices becoming available at scale.

\section{ACKNOWLEDGMENT}

The authors would like to thank the SHL Group AB for funding and access to their laser diffraction measurement setup.

\section{REFERENCES}

[1] H. H. Kyu et al., "Global, regional, and national disability-adjusted lifeyears (DALYs) for 359 diseases and injuries and healthy life expectancy (HALE) for 195 countries and territories, 1990-2017," Lancet, vol. 392, pp. 1859-1922, Nov. 2018.

[2] Findings From the Global Burden of Disease Study 2017, IMHE, Seattle, WA, USA, 2018.

[3] S. P. Newman, "Inhaler treatment options in COPD," Eur. Respiratory Rev., vol. 14, no. 96, pp. 102-108, Dec. 2005.

[4] J. B. Fink, G. L. Colice, and R. Hodder, "Inhaler devices for patients with COPD," COPD: J. Chronic Obstructive Pulmonary Disease, vol. 10, no. 4, pp. 523-535, Aug. 2013.

[5] W. Van Hoeve, S. Gekle, J. H. Snoeijer, M. Versluis, M. P. Brenner, and D. Lohse, "Breakup of diminutive Rayleigh jets," Phys. Fluids, vol. 22, no. 12, Dec. 2010, Art. no. 122003.

[6] H. W. Frijlink and A. H. De Boer, "Trends in the technology-driven development of new inhalation devices," Drug Discovery Today: technol., vol. 2, no. 1, pp. 47-57, Mar. 2005.

[7] A. H. De Boer et al., "In vitro performance testing of the novel medspray wet aerosol inhaler based on the principle of Rayleigh break-up," Pharm Res, vol. 25, no. 5, pp. 1186-1192, May 2008.

[8] S. P. Newman, "Principles of metered-dose inhaler design," Respiratory Care, vol. 50, no. 9, pp. 1177-1190, Sep. 2005.
[9] R. Dalby, M. Spallek, and T. Voshaar, "A review of the development of Respimat soft mist inhaler," Int. J. Pharmaceutics, vol. 283, nos. 1-2, pp. 1-9, Sep. 2004.

[10] H. Heijerman, E. Westerman, S. Conway, and D. Touw, "Inhaled medication and inhalation devices for lung disease in patients with cystic fibrosis: A European consensus," J. Cystic Fibrosis, vol. 8, no. 5, pp. 295-315, Sep. 2009.

[11] A. H. De Boer, P. Hagedoorn, M. Hoppentocht, F. Buttini, F. Grasmeijer, and H. W. Frijlink, "Dry powder inhalation: Past, present and future," Expert Opinion Drug Del., vol. 14, no. 4, pp. 499-512, Apr. 2017.

[12] T. S. Last, G. Stemme, and N. Roxhed, "Demonstration of the first self-sealing aerosol spray nozzle for medical drug delivery," in Proc. IEEE 32nd Int. Conf. Micro Electro Mech. Syst. (MEMS), Jan. 2019, pp. 53-56.

[13] J. Mannik, R. Driessen, P. Galajda, J. E. Keymer, and C. Dekker, "Bacterial growth and motility in sub-micron constrictions," Proc. Nat. Acad. Sci. USA, vol. 106, no. 35, pp. 14861-14866, Sep. 2009.

[14] S. Waterhouse and G. Hall, "The validation of sterilising grade microfiltration membranes with Pseudomonas diminuta a review," J. Membrane Sci., vol. 104, nos. 1-2, pp. 1-9, Aug. 1995.

[15] H. Kim and K. Najafi, "Wafer bonding using parylene and waferlevel transfer of free-standing parylene membranes," presented at the Transducers Conf., Boston, MA, USA, 2003.

[16] H. Kim and K. Najafi, "Characterization of low-temperature wafer bonding using thin-film parylene," J. Microelectromech. Syst., vol. 14, no. 6, pp. 1347-1355, Dec. 2005.

[17] H.-S. Noh, K.-S. Moon, A. Cannon, P. J. Hesketh, and C. P. Wong, "Wafer bonding using microwave heating of parylene intermediate layers," J. Micromech. Microeng., vol. 14, no. 4, pp. 625-631, Apr. 2004.

[18] B. J. Kim and E. Meng, "Micromachining of parylene C for bioMEMS," Polym. Adv. Technol., vol. 27, no. 5, pp. 564-576, May 2016.

[19] S. P. Timoshenko and S. Woinowsky-Krieger, Theory of Plates and Shells. New York, NY, USA: McGraw-Hill, 1987.

[20] J. Eggers and T. F. Dupont, "Drop formation in a one-dimensional approximation of the Navier-Stokes equation," J. Fluid Mech., vol. 262, pp. 205-221, Oct. 1994.

[21] D. S. Viswanath and T. K. Ghosh, Viscosity of Liquids. Dordrecht, The Netherlands: Springer, 2007.

[22] M. A. Hopcroft, W. D. Nix, and T. W. Kenny, "What is the young's modulus of silicon?" J. Microelectromech. Syst., vol. 19, no. 2, pp. 229-238, Apr. 2010.

[23] C. Van Rijn, M. Van Der Wekken, W. Nijdam, and M. Elwenspoek, "Deflection and maximum load of microfiltration membrane sieves made with silicon micromachining," J. Microelectromech. Syst., vol. 6, no. 1, pp. 48-54, Mar. 1997.

[24] K. E. Petersen, "Silicon as a mechanical material," Proc. IEEE, vol. 70, no. 5, pp. 420-457, May 1982.

[25] H. Lee, D. Ham, and R. M. Westervelt, Eds., CMOS Biotechnology. New York, NY, USA: Springer, 2007.

[26] Z. Dagan, S. Weinbaum, and R. Pfeffer, "An infinite-series solution for the creeping motion through an orifice of finite length," J. Fluid Mech., vol. 115 , no. 1, p. 505, Feb. 1982.

[27] A. R. Clark, "The use of laser diffraction for the evaluation of the aerosol clouds generated by medical nebulizers," Int. J. Pharmaceutics, vol. 115, no. 1, pp. 69-78, Feb. 1995.

[28] P. Kippax, "Appraisal of the laser diffraction particle-sizing," Pharmaceutical Technol., vol. 17, pp. 32-39, Jan. 2005.

[29] T. Allen, Particle Size Measurement. Dordrecht, The Netherlands: Springer, 1990.

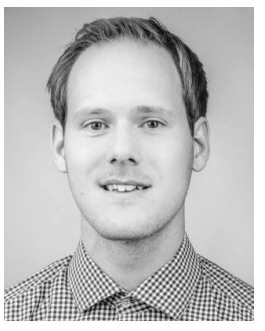

Torben Sebastian Last received the M.Sc. degree in nanotechnology from Leibniz Universität Hannover, Germany. He is currently pursuing the Ph.D. degree in micro and nanosystems with KTH Royal Institute of Technology, with a focus on liquid-jet drug delivery devices. 


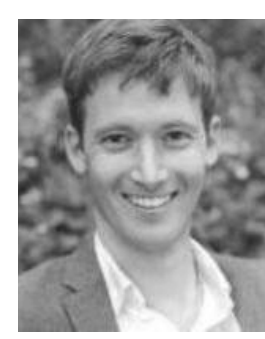

Niclas Roxhed received the M.Sc. degree in electrical engineering and the Ph.D. degree in microsystem technology from KTH Royal Institute of Technology, Stockholm, Sweden, in 2003 and 2007, respectively. $\mathrm{He}$ is currently an Associate Professor and the Team Leader of medical MEMS with KTH and a Research Fellow with the Langer Laboratory, Massachusetts Institute of Technology (MIT), Boston, MA, USA. He has authored or coauthored more than 100 scientific articles and 29 patent applications. His main research fields are sensors for medical diagnostics, MEMS-based drug delivery, and medical-aid microsystems.

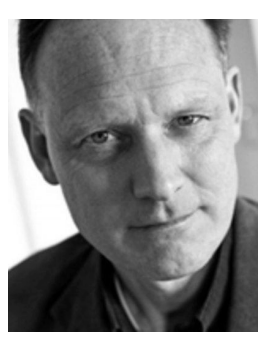

Göran Stemme (Fellow, IEEE) received the M.Sc. degree in electrical engineering and the Ph.D. degree in solid-state electronics from the Chalmers University of Technology, Gothenburg, Sweden, in 1981 and 1987, respectively. In 1981, he joined the Department of Solid State Electronics, Chalmers University of Technology, where he became an Associate Professor (docent) heading the Silicon Sensor Research Group in 1990. Since 1991, he has been a Professor with KTH Royal Institute of Technology, Stockholm, Sweden, where he is also the Head of the Department of Micro and Nanosystems, School of Electrical Engineering. His research on micro and nanotechnology and systems spans over a broad range of techniques and application fields, such as medical technology, microfluidics, optical applications, wafer-level packaging, and device integration. He has published more than 360 research journal and conference papers. His publications have more than 8500 citations and an H-index of 48 according to Scopus. He is also a member of the Royal Swedish Academy of Sciences (KVA) and the Royal Swedish Academy of Engineering Sciences (IVA). 